Théologiques

Théologiques

\title{
Charisme et souffrance de l'éditeur politique : François Maspero
}

\section{Camille Joseph}

Volume 17, numéro 1, 2009

Les charismes

URI : https://id.erudit.org/iderudit/039499ar

DOI : https://doi.org/10.7202/039499ar

Aller au sommaire du numéro

\section{Éditeur(s)}

Faculté de théologie et de sciences des religions, Université de Montréal

ISSN

1188-7109 (imprimé)

1492-1413 (numérique)

Découvrir la revue

Citer cet article

Joseph, C. (2009). Charisme et souffrance de l'éditeur politique : François Maspero. Théologiques, 17(1), 79-93. https://doi.org/10.7202/039499ar
Résumé de l'article

Centré sur le personnage de l'éditeur français François Maspero, cet article traite des liens entre le charisme et la souffrance de l'individu charismatique. Il met en perspective d'un côté l'existence et la manifestation de la domination charismatique dans le contexte politique des années 1960 et 1970 et, de l'autre côté, l'investissement personnel extrêmement appuyé dans le travail, débouchant sur un rapport douloureux et contraignant à celui-ci. Loin d'être la conséquence d'un mode d'exercice du métier, la souffrance doit plutôt être rapportée aux motivations biographiques et à l'héritage familial à l'origine du choix de l'investissement dans un métier, l'édition, sur un modèle contestataire. 
Théologiques 17, no 1 (2009) p. 79-93

\title{
Charisme et souffrance de l'éditeur politique: François Maspero
}

\author{
Camille JOSEPH* \\ Sociologie \\ École des hautes études en sciences sociales, Paris
}

Les éditions Maspero furent créées à Paris en 1959. Leur fondateur, François Maspero, avait d'abord tenu une librairie qu'il avait achetée quelques années auparavant, La Joie de lire. Devant le silence des maisons d'édition françaises face aux crimes de la guerre d'Algérie, auquel faisaient seulement exception les éditions de Minuit dirigées par Jérôme Lindon, François Maspero décida de publier, entre autres, les livres des théoriciens de la décolonisation et des auteurs issus des pays en lutte du Tiers monde (Simonin 1991; Liauzu 1991). Parmi les premiers titres, on trouve L'an V de la révolution algérienne, de Frantz Fanon (1959), Le refus de Maurice Maschino (1960) ou encore Aden Arabie de Paul Nizan (1960). Parus dans la fameuse collection "Cahiers libres ", ces livres incarnent l'engagement politique qui marqua l'image de la maison d'édition. Cet engagement participa à la construction d'une figure quasi héroïque au sein de l'édition française et d'un modèle d'édition politique qui continue d'être une référence prestigieuse aujourd'hui encore.

Mais le projet politique des éditions animées par François Maspero ne se résumait pas tout entier dans la teneur des livres publiés. L'éditeur tenait à mettre le mode de fonctionnement de l'entreprise au diapason des conceptions politiques qui étaient les siennes. Malgré son statut «classique ", l'entreprise fut le cadre de l'expérimentation d'un projet de gestion non commercial, non soumis à un impératif de rentabilité et, autant que possible, non hiérarchique. Les archives de la maison révèlent les efforts répétés de François

* Camille Joseph est doctorante au Centre de sociologie européenne (EHESS, Paris). Elle termine actuellement une thèse de sociologie intitulée: Un héritage éditorial: les éditions La Découverte, sous la direction de Gisèle Sapiro. Elle a récemment publié «Les éditions Maspero et les éditions La Découverte face à l' “étranger” ", dans G. Sapiro, dir., Les contradictions de la globalisation éditoriale, Paris, Nouveau Monde, 2009. 
Maspero pour ne pas apparaître comme un patron comme les autres, ainsi que les tentatives de mise en place d'un "patron collectif ${ }^{1}$ ». À ce titre, François Maspero fut doublement un éditeur politique en vertu, à la fois, de la nature subversive des livres publiés et du caractère révolutionnaire du projet éditorial lui-même.

Cependant, en dépit des différentes mesures destinées à organiser le fonctionnement de l'entreprise sur un mode (relativement) collectif, le directeur de la maison conserva l'essentiel des responsabilités économiques, pénales et éditoriales. Il fut non seulement confronté aux échéances des fins de mois et aux différents procès qui lui furent intentés, mais il fut également «maître d'œuvre et maître de son œuvre ${ }^{2}$ ». Sa position fut celle d'un individu aux qualités reconnues comme «extraordinaires ", exerçant, sur l'ensemble des salariés de sa maison, une forme d'autorité spécifique qui se manifesta par le dévouement de ces derniers à la «cause» révolutionnaire des éditions et par le prestige recueilli par l'éditeur au fil du temps: le charisme.

Dans des documents écrits par l'éditeur, on note la présence d'une troisième dimension, à côté de l'engagement politique et du charisme: la souffrance. Sous sa plume sont en effet dévoilées les conséquences douloureuses liées à son investissement intense dans le métier d'éditeur. Dans les archives de la maison, de nombreux textes, rédigés entre 1969 et 1975, mentionnent de façon explicite les épisodes de fatigue, voire les expériences plus éprouvantes qui frappèrent l'éditeur. François Maspero emprunte le registre de la souffrance et de la pénibilité pour décrire son métier, avec une insistance que l'on rencontre rarement chez d'autres éditeurs. Ces documents côtoient d'autres textes, eux aussi rédigés et signés à la première personne. Qu'ils concernent le projet éditorial ou des questions de gestion quotidienne, ils n'avaient de cesse de rappeler ou d'améliorer les visées politiques de la maison.

1. Cette expression a été utilisée par Charles Péguy: «[c]e que je nomme un patron collectif était, si vous le voulez bien, le conseil d'administration [...]». (1947, 47)

2. C'est dans un texte saisissant publié en 2002 que François Maspero se définit luimême ainsi, reprenant l'interprétation particulière de la position de "patron » qu'il avait employée ailleurs pour qualifier le travail du cinéaste Chris Marker. Ce dernier réalisa, en 1970, un film sur François Maspero: On vous parle de Paris. Maspero: Les mots ont un sens. À propos de ce film, François Maspero écrivait: «Au tournage, Chris Marker avait tenu compte de toutes les suggestions, de toutes les initiatives. Les idées de chacun étaient prises en compte de façon égale. [...] Dans toute cette diversité que je viens de dire, Chris Marker, maître d'œuvre et maître de son œuvre a fait un choix $[\ldots] » .(2002,261)$ 
Dans cet article, je me propose d'interroger le lien entre charisme, édition politique et souffrance personnelle. La dimension politique du métier d'éditeur tel que l'a investi François Maspero et le charisme individuel de ce dernier constituent deux éléments $a$ priori discordants: le succès à titre personnel de l'éditeur contredit la dimension révolutionnaire du projet éditorial et en particulier le désir d'un collectif. Il s'agira d'abord de situer la souffrance dans cette économie de la domination charismatique. Dans un deuxième temps, loin de m'arrêter à l'idée que cette souffrance serait la conséquence «logique » d'un investissement extraordinaire dans le métier, je montrerai que la souffrance trahit surtout un rapport ambivalent à l'histoire familiale qui ne pouvait se résoudre dans un rapport «facile» à l'exercice du métier d'éditeur et encore moins à la position charismatique.

\section{Au nom de la révolution}

Loin d'être tenue pour secrète, la souffrance se manifeste, sous la plume de François Maspero, dans de nombreux documents d'archives. Une partie de ces textes sont des documents adressés à l'ensemble des salariés de la librairie et des éditions. D'autres, toujours signés de la main de François Maspero lui-même, visaient à informer les auteurs et les lecteurs. Dans les sources disponibles, qui varient à la fois dans leur forme et dans leur degré de confidentialité, la difficulté de faire face au travail et la pénibilité de ce dernier sont énoncées sous une forme plus ou moins euphémisée. Ainsi, par exemple, on peut lire dans un document datant de 1971:

J'ai travaillé avec acharnement. [...] Ne gagnant que mon salaire, ne recueillant aucun des profits dus à ma «situation sociale» mais bien tous les emmerdements, travaillant le double ou le triple des autres, seul responsable civilement et pénalement, seul à avoir conscience des menaces véritables qui pèsent sur la sécurité de notre emploi à tous, j'ai cru pouvoir demander que l'on ait à mon égard le minimum de respect que je garantissais aux autres membres du personnel ${ }^{3}$.

À travers cette description, François Maspero se situe «seul» face aux "autres ", à la fois sur le plan de la responsabilité pénale, de la gestion et du travail en général. Mais il ne se distingue plus dès lors que l'on considère le salaire ou d'autres types de "profits ». Contre l'idée d'un patron qui profiterait sur le dos des travailleurs, l'éditeur déclare que ses seuls privilèges

3. Document daté du 27 mars 1971 dans Archives La Découverte. 
se mesurent en termes de difficultés, de responsabilités, de fatigue et de souffrance.

Dès lors, la souffrance exprimée dans cet extrait, comme dans les autres textes, n'est pas affichée pour elle-même. Elle est un argument opposé aux salariés, notamment ceux de la librairie, qui l'accusaient d'exercer une forme de paternalisme inacceptable et de faire du profit. Dans le contexte politique et social des années 1960 et 1970, Maspero était en effet constamment à l'affût de la moindre critique relative à un quelconque statut privilégié, que ce soit celui de "patron» ou de «bourgeois ». Il n'avait de cesse de vouloir prouver qu'il était fils de bourgeois, certes, mais un «bourgeois qui trahit la bourgeoisie» (Marker 1971). Dans le cadre d'une certaine tentative d'émancipation par rapport au modèle patron/travailleur, dénoncé comme une aliénation, l'individualisation des fonctions de directeur posait alors problème.

François Maspero tenta ainsi de bousculer la hiérarchie interne de la maison en mettant en place, en 1969, un directoire, c'est-à-dire une instance de direction à vocation collective. L'existence de cette structure collective entraîna, dans les années qui suivirent, la production accélérée de textes explicatifs et justificatifs destinés à régler les rapports entre les personnes impliquées dans l'entreprise. Loin de favoriser une répartition plus juste des tâches, la décision de passer à une société à directoire provoqua l'émergence de nouvelles difficultés qui se traduisirent par l'épuisement progressif de l'éditeur. Il écrivait ainsi qu'il était «bloqué devant un véritable mur» (dans un compte rendu de séance du directoire daté d'octobre 1970) et, jusqu'au milieu des années 1970, il traversa une crise personnelle profonde (2002, 254-255).

De façon paradoxale, le prestige grandissant des éditions Maspero fut corrélé à une absence de reconnaissance de la quantité de travail très importante fournie par l'éditeur (et par les autres salariés de l'entreprise). Le prestige personnel que François Maspero recueillait était souvent confondu avec une position privilégiée à tous les points de vue. Jusqu'au milieu des années 1970, il paraissait en effet surmonter toutes les épreuves (financières, éditoriales, pénales) avec un relatif «succès ", c'est-à-dire qu'il maintenait l'existence de sa maison, même si le déficit, en 1970 encore, ne fut résorbé que grâce aux bénéfices modestes de la librairie. Charles Péguy avait déjà remarqué, avec autant de surprise et non sans un certain dépit, que les regards extérieurs posés sur les Cahiers de la quinzaine pouvaient voir en eux une entreprise fonctionnant bien et ne connaissant pas de difficultés particulières. 
Les Cahiers ayant l'apparence du succès et de la prospérité, Charles Péguy estimait nécessaire de rappeler régulièrement que cette réussite reposait sur un engagement sans faille et sur l'ardeur de leur fondateur:

Nous souffrons surtout, depuis quatre ou cinq ans, d'une opinion totalement fausse qui s'est établie sur la prospérité de nos cahiers. Quand nous rencontrons des gens, même des amis, qui ne nous ont pas vus depuis un certain temps: Eh bien! nous disent-ils, ça va très bien les cahiers. Ils veulent dire par là confusément ensemble: qu'ils sont très contents des cahiers qu'ils reçoivent, qu'ils entendent parler très favorablement partout des cahiers, et que par conséquent nous sommes évidemment très à l'aise pour les fabriquer. [...] Qu'ils se détrompent. Et qu'ils se détrompent doublement. Qu'ils se détrompent en fait. Si en fait en ce temps de sabotage universel nous avons maintenu la décence et la propreté de la fabrication, de toutes les fabrications, de la fabrication intellectuelle et de la fabrication industrielle, de la plume et de l'encre, de la typographie et de la copie, du papier et de l'œuvre, ce n'est point que nous en ayons ni que nous en ayons jamais eu les moyens, c'est que depuis quinze ans nous travaillons très au-dessus de nos moyens. Nous ne sommes à aucun degré ni en aucun sens des amateurs. Nous sommes dans le sens le plus rigoureux de ce mot, le plus beau de tous, des professionnels. (Péguy 1947, 272-273)

On trouve dans les archives de la maison d'édition Maspero des mises au point similaires, quoique moins virulentes peut-être; ainsi ce long document de seize pages intitulé "Sur le rôle de la librairie Maspero et de ceux qui y travaillent ", daté de septembre 1971, dans lequel François Maspero reprend et développe les points d'assise du projet de l'entreprise, à la fois économique, politique et social. Dans le long extrait cité, Charles Péguy manifeste la nécessité de montrer ce qui reste caché: le "prix » humain du travail. Le temps et l'énergie investis dans le travail éditorial ne sont en effet pas vécus sur un mode silencieux: les éditeurs râlent, se plaignent, manifestent leur souffrance. Leur travail, dont ils ont une haute idée, requiert un degré fort d'investissement. Cela devient une marque de "professionnalisme ", et est loin d'être vécu comme un "sacrifice». Dans ce contexte, l'ostentation de la "souffrance» n'a pas pour but de réclamer un regard plus compatissant sur soi. Elle vise à dénoncer le raisonnement logique qui, du constat de la qualité des livres, conclut à la prospérité de l'éditeur. L'investissement personnel très important de l'éditeur dans son travail n'est pas seulement indexé sur la qualité des livres publiés. Celle-ci est plus admirable encore car elle est associée à un projet politique révolutionnaire dénonçant l'ordre établi et se distinguant d'un projet éditorial «classique», celui d'une entreprise capitaliste quelconque. 
Dans l'extrait cité plus haut, François Maspero rappelle qu'il est «seul responsable civilement et pénalement, seul à avoir conscience des menaces véritables qui pèsent sur la sécurité de notre emploi à tous ". En tant que propriétaire de la maison d'édition, seul actionnaire puis, à partir de 1969, président du directoire, il est effectivement seul à assumer les responsabilités juridiques et économiques. À côté de ces fonctions, François Maspero prend également les décisions éditoriales et assure le travail réservé à l'éditeur dans les relations avec les fournisseurs et les auteurs. L'intensité extrême de cet investissement dans le travail est justifiée par les arguments suivants: situation économique (mauvaise) de l'entreprise, qui empêcherait par exemple d'embaucher et de soulager le «patron»; position (dominée) parmi les autres maisons d'édition, c'est-à-dire un accès aux circuits de diffusion ou de distribution plus difficile que pour les maisons installées et une position fragilisée par les atermoiements judiciaires; motivations politiques enfin, car l'entreprise n'est au service ni du patron ni de l'actionnaire mais seulement à celui du projet militant.

La position particulièrement difficile de la maison, en termes d'assise sociale et économique, réclamait un investissement absolu dans le travail, qui était lui-même légitime politiquement. Peu solide économiquement, ne bénéficiant pas non plus de "gages» de prestige durable, c'est-à-dire du capital inscrit dans le nom de quelques best-sellers, la maison d'édition était, à l'image de son fondateur, en position de «traître " face à "l'édition bourgeoise» (incarnée par exemple par les éditions Gallimard):

Je n'étais lié à aucune formation politique. Plus grave, je n'avais moi-même aucune formation tout court, ni expérience, ni diplômes. Aucune légitimité, pas même celle de l'argent, qui rassure le bourgeois et encore plus, quoi qu'ils en disent, ceux qui prétendent ne pas l'être. Je m'étais arrogé la liberté d'éditer ce qui aurait pu être n'importe quoi sur ces sujets auxquels je ne connaissais rien. (Maspero 2002, 189)

Ce paradoxe constitutif du projet éditorial, intellectuel et politique de François Maspero - trahir la bourgeoisie avec ses propres armes — s'incarne dans les livres publiés par les éditions, investis de tout le savoir-faire, le professionnalisme, l'attention et le goût de l'éditeur. Le travail à fournir est double: il faut produire de beaux et bons livres, et il faut les produire sans les outils techniques, financiers ou humains de la grande édition. C'est ce que disait déjà Péguy dans l'extrait cité plus haut: la réalisation d'un cahier qui a toute «la décence et la propreté » de l'édition de qualité (de la typographie à la qualité du papier) demande de «travail[ler] très au-dessus 
de nos moyens". La souffrance, conséquence d'un investissement exceptionnel, devient dès lors la marque du travail véritable, contrastant avec le relatif confort de l'édition bourgeoise; au modèle d'un rentier d'édition vient ainsi s'opposer celui de l'artisan. Cette figure est extrêmement valorisée car elle est associée à un travail plus manuel, donc plus prestigieux du point de vue des catégories politiques de l'éditeur (proximité avec la figure de l'ouvrier ${ }^{4}$ ). La souffrance se situe du côté des artisans, des ouvriers, des "petits "; il faut la dire, toujours, pour écarter l'idée selon laquelle ces «beaux» livres seraient produits sans peine grâce aux revenus du capital. Alors apparaît l'objet digne d'une admiration toute spéciale: le livre politique paré des atours de l'édition bourgeoise grâce au travail acharné de l'éditeur.

Travailler comme un "artisan", créer sans capitaux, tout comme devenir éditeur sans avoir suivi de formation, permet d'obtenir un grand "crédit » aux yeux des militants suspicieux de la moindre trace d'héritage bourgeois. Créer sans l'assurance du lendemain, c'est développer l'image d'un virtuose de la pénurie contre celle d'un héritier à qui tout est acquis. Le caractère anti-économique de la maison d'édition "force» l'éditeur à user de ses qualités pour l'empêcher de disparaître. Ce que l'on ne prend pas à l'argent, Péguy le rappelle, «il faut toujours que cela se prenne quelque part, et que ce qui ne coûte pas à l'argent, ce qui ne coûte pas en argent, se paie par un surmenage perpétuel, un risque de mort constant, des ravages irréparables pour la santé » $(1947,275)$. La souffrance apparaît alors comme la conséquence du travail réclamé par une maison d'édition étrangère au profit économique et obéissant à une finalité politique. Légitimée par le caractère révolutionnaire du projet éditorial, elle peut ainsi exister sans que son porteur puisse trouver en elle un motif de distinction ou de valorisation personnelle particulière (en attirant par exemple à lui la compassion). Susceptible d'appeler une dévotion totale, la cause politique rend légitime la pénibilité du travail et l'auto-exploitation totale de l'individu. Cette position est exprimée de façon radicale sous la plume d'un autre éditeur militant, Maurice Hasfeld, gérant de la Librairie du travail, à Paris, dans les années 1920: «la Librairie est ma passion et je n'attends d'elle que des

4. En tant qu'intellectuels, les éditeurs tendent à dénier la place du corps dans le travail et seule la revendication d'un point de vue d' "artisan" les autorise à exprimer leur fatigue physique. La récurrence, dans les archives, de l'évocation de faiblesses physiques (autant que morales) demanderait à ce que soient explorés les enjeux souvent oubliés et pourtant décisifs associés au corps, corps malade et fatigué, dans une sociologie du métier d'éditeur et des métiers intellectuels en général. 
soucis. Je n'attends aucune reconnaissance ni aucun compliment. Mais je ne veux pas non plus d'article nécrologique sur mon dévouement et mon désintéressement. [...] Le mouvement ouvrier a besoin de certaines choses et c'est pour lui qu'il faut travailler, c'est pour lui qu'il faut soutenir toute initiative susceptible de lui être utile ${ }^{5}$ (Bardouillet 1977, 171).

Si la souffrance est montrée comme la conséquence inévitable du caractère politique du projet éditorial, son évocation régulière doit être mise en relation avec l'émergence d'un personnage charismatique. On l'a dit, l'ostentation de la souffrance n'a pas pour but de provoquer la compassion du groupe. Pourquoi doit-elle alors se manifester de manière visible (ou audible) ? Comme l'a montré Max Weber, l'acquisition du charisme par un individu suppose nécessairement un passage par des étapes douloureuses. La douleur vient marquer, dans l'épreuve initiatique, le passage à un autre état, voire à un autre moi ${ }^{6}$. Max Weber écrit: «le héros doit [...] renaître pour accéder à l'hérö̈sme. L'éducation charismatique en ce senslà, avec ses noviciats, ses épreuves de courage, ses tortures, ses degrés d'initiation et de dignité, ses initiations des jeunes et ses prises d'armes, est une institution universelle, commune à toute socialisation guerrière, dont il subsiste presque partout des vestiges » (Weber 2006, 186-187). Si l'apprenti charismatique ne souffre pas directement devant ceux qui reconnaissent en lui un "chef », il fait le récit des épreuves subies ou il en porte la trace visible. Comme le rappelle Pierre Clastres, c'est la marque laissée par la souffrance initiatique - la balafre, la cicatrice, la blessure - qui atteste le succès de l'initiation. L'ostentation de la souffrance, plus que la souffrance elle-même, est loin de correspondre à l'aveu impudique d'une pulsion masochiste. La douleur, vécue comme telle, s'incruste dans le corps et, suggère Pierre Clastres, ramène l'individu à la société tandis que cette dernière peut

5. Né en 1889, Marcel Hasfeld, syndicaliste et militant communiste, fut administrateur de la revue La vie ouvrière avant de devenir le gérant de la Librairie du travail, créée le 11 novembre 1917. Ce fut d'abord une bibliothèque qui se donna pour objectif de diffuser la culture aux ouvriers. En 1928, elle fut transformée en une coopérative ouvrière d'édition. Les problèmes financiers s'accumulant, le stock de la Librairie fut vendu aux enchères en 1939.

6. Pour Max Weber, les compétences extraquotidiennes, inscrites «en germe» dans le corps, sont "réveillées" par un ensemble de pratiques ascétiques et douloureuses: «[...] Bien qu'il soit impossible d'éveiller les facultés charismatiques dans un objet ou chez une personne qui ne les possèdent pas en germe, ce germe reste caché si on ne l'amène pas à se développer, si on ne "réveille" pas le charisme - par l' "ascèse" par exemple» (2006, 81-82). L'initiation vise donc à transfigurer un ensemble de qualités extraordinaires en une somme de pouvoirs positifs. 
à son tour se reconnaître dans un de ses corps. L'individu doit montrer que cette marque a été incrustée dans son corps: «La marque est un obstacle à l'oubli, le corps lui-même porte imprimées sur soi les traces du souvenir, le corps est une mémoire» (Clastres 1974, 157). La souffrance et son ostentation viennent apporter, aux yeux du groupe, la preuve de l'initiation, c'est-à-dire du fait que l'individu possède des qualités extra-ordinaires (Kalinowski 2005, 117 sq.). La souffrance donne alors au groupe la «confiance» d'où peut naître l' "abandon» dans la croyance. Le charisme peut se définir comme un héroïsme de la souffrance, dans lequel celui qui souffre ou a souffert est devenu celui qui «brille».

Le charisme, qui repose sur la croyance dans un message révolutionnaire incarné dans un individu, puise là la force de son autorité. Ainsi, dans le cas de François Maspero, l' " autorité charismatique » de l'éditeur ne dérivait pas entièrement de sa position dominante de directeur, qu'il faudrait plutôt identifier, selon le modèle wébérien, à une forme d'autorité bureaucratique $^{7}$. Mais ces deux autorités peuvent se confondre en un seul individu. François Maspero tenta, on l'a dit, de contester à lui-même les privilèges de la position de "patron ", sans succès. Ses efforts en ce sens s'avérèrent relativement vains. Cet échec du collectivisme, Charles Péguy l'avait lui aussi expérimenté. Il avait constaté de façon lucide combien il était difficile d'allier un projet de coopérative de travailleurs et la personnalité d'un «homme impossible", selon ses propres termes, qui ne supportait pas de n'être pas le chef du groupe en question (Noiriel 2005, 72). D'une part, l'investissement radical dans son métier hissa l'éditeur au rang de quasi-«héros». D'autre part, cet «individu» individualisé par son projet, notamment, tenait non pas un discours légitimant sa propre position mais, au contraire, un discours révolutionnaire qui visait à renverser l'ordre établi (celui qu'il incarnait paradoxalement). Ces deux éléments participaient à la croyance dans les qualités extraordinaires de l'éditeur. Mais l'éditeur ne pouvait pas tolérer, et le groupe charismatique non plus, d'obtenir un succès individuel sur les bases d'un projet anti-capitaliste censé dénoncer toutes les formes de domination. Dans le cadre du projet militant, le prestige d'un seul, l'éditeur, ne pouvait pas être reconnu en tant que tel et faire, de manière "officielle", l'objet de la dévotion toute particulière du groupe charismatique. La souffrance personnelle, l'investissement total, l'engagement complet de la personne de François Maspero au service du projet politique et éditorial

7. Sur les trois formes de domination, légale, traditionnelle et charismatique, voir Weber (1995 [1971], 289 sq.). 
avaient pour fonction de relier les deux dimensions contradictoires que constituaient le projet et la position de directeur. La souffrance devient l'élément résolutoire de cette contradiction.

\section{L'épreuve permanente}

L'insistance dans l'exhibition de la souffrance, pendant de très nombreuses années, suggère que celle-ci occupait une place plus précise dans le rapport de François Maspero à son travail. On l'a dit, elle ne servait pas à appeler à l'aide ni à demander que soit mis fin à une situation qui était par ailleurs qualifiée d' «injuste». Dans l'extrait cité plus haut, François Maspero réclamait plutôt du «respect » et la reconnaissance du travail honnête et bien fait. Son désir profond était de ne pas être confondu avec le directeur capitaliste et fainéant d'une maison d'édition bourgeoise. Bourgeois, il l'était pourtant par héritage familial, et, comme il le disait lui-même, il ne pouvait donc penser sa position au même niveau que celle des non-bourgeois, des militants, de certains auteurs de la maison, etc. Celle-ci pouvait même être difficile à tenir :

Naturellement, des camarades que j'estimais me disaient que j'étais plus utile à ma place, comme sirène d'alarme, caisse de résonance, relais, courroie de transmission et autres métaphores [...]. Mais, pour moi, il restait que si mon travail était un combat, ce combat était petit, souvent confus et contradictoire, et que même s'il était quotidien et difficile, il ne comportait pas les risques que prenaient certains de ceux dont je répercutais les voix. [...] La question plus pertinente était: si j'abandonnais ce métier dont j'étais quand même convaincu que je le faisais utilement, étais-je sûr que je serais plus utile - et plus heureux - ailleurs? Le fait est que j’ai continué métier et profession, et que je n'ai guère réussi, ces années-là, à me rendre à cette injonction d'être pleinement heureux. (Maspero 2002, 254)

À l'opposition entre «moi, tout seul» et «les autres » vient s'ajouter une autre rupture, celle qui sépare celui qui mène un combat «petit », "confus et contradictoire » face à ceux qui, on le devine en négatif, luttent «en grand », les militants et les résistants. Bien qu'il reconnaisse le danger objectif lié à son propre engagement contre la guerre d'Algérie, par exemple, et les risques inhérents à la publication de livres condamnés et interdits, François Maspero livre dans cet extrait son propre jugement à l'égard de son travail. Mesurant ses difficultés, et donc sa souffrance, à l'aune du combat mené par les militants, l'éditeur se fait le pire critique de son ancien métier, métier d'«intellectuel », métier passif, etc. Il a dit qu'il avait été passionné par 
l'édition et il est certain que les choix esthétiques incarnés dans ses livres témoignent d'un tel engagement; néanmoins, il s'est senti incapable de mettre la valeur politique de son travail sur le même plan que celle d'un résistant ou d'un militant «de terrain». Ce point de vue ne se confond pas avec la rhétorique consistant à dénier aux livres leur qualité d' "armes ». Mais il n'en produit pas moins un certain discours de dévalorisation. La souffrance du "privilégié » est toujours plus "petite» que la souffrance des militants qui luttent et qui risquent leur vie.

Celui qui s'est «le plus» battu et qui a «le plus» souffert, d'une certaine manière, c'est Jean, son frère ${ }^{8}$. Né en 1925 , jeune militant communiste, Jean entra en résistance très tôt et mène parfois des actions en compagnie de son petit frère François. Brillant élève, promis à l'École normale supérieure, ce grand frère semblait avoir reçu les galons de digne héritier d'une famille d'universitaires de renom (le grand-père Gaston fut un égyptologue consacré; le père, Henri, était un sinologue non moins renommé, professeur au Collège de France). Soupçonnés par les Allemands d'activités terroristes, Henri et Hélène Maspero furent déportés en 1944. Tandis que la mère des enfants revint vivante du camp de Ravensbrück, le père mourut à Buchenwald et Jean fut tué au combat en Moselle. La résistance et le militantisme ont donc été une affaire de famille et il est certain que François Maspero a toujours, d'une part, souhaité s'en montrer digne et, d'autre part, toujours douté de sa capacité à l'être réellement. Dès lors, il est vrai, la douleur de ces deuils et le devoir d'honorer les résistants forment la base de son engagement politique. L'édition fut le lieu de la poursuite de cette tradition, au prix d'un investissement financier (l'héritage de sa grand-mère permit d'acheter la librairie), physique et psychique très important. Mais cela n'empêche pas le doute récurrent quant au «mérite» et au «droit de vivre» du survivant:

Mais aujourd'hui je vois bien qu'à la source de cette vague culpabilité qui colle à l'esprit comme à la peau, qui colle à toute une vie et qu'aucune rationalité ne peut effacer, il y a autre chose: ce n'est pas tout d'être un survivant, encore faudrait-il pouvoir se prouver qu'on en est digne. Qu'on n'est pas seulement un laissé-pour-compte. Et je ne suis pas de ceux qui pensent s'en

8. Pour se convaincre du traumatisme laissé par la mort de Jean, il suffit de lire une interview récente de François Maspero qui lui était tout entière consacrée. L'article est accompagné d'un photomontage où la moitié du visage de François Maspero est complétée par un dessin figurant l'absent, souriant (Aeschimann 2007). 
tirer par l'exercice de "devoir de mémoire» — même si je ne mets pas en cause son utilité, car que fais-je d'autre en ce moment que de l'exercer? (Maspero 2002, 67)

François Maspero semble toujours placer la dignité morale et le courage politique du côté de son frère; à lui-même, il réserve les doutes associés, du point de vue des "purs» intellectuels, aux activités de commerce. L'édition et la gestion d'une librairie mêlent précisément des intérêts intellectuels, désintéressés, et des intérêts économiques. La présence de ces derniers confère au métier d'éditeur un goût de «trahison " par rapport à la carrière universitaire à laquelle Jean semblait destiné. François Maspero écrit: «je doute que Jean eût approuvé le choix de son petit frère de faire carrière derrière un tiroir-caisse » (190).

On a alors raison de se demander quel aspect du métier d'éditeur est le plus honteux. Il n'est pas certain que la dimension économique de l'édition ne soit pas en vérité une plus grande source de dévalorisation et d'autodépréciation que la dimension politiquement "passive » ou éclectique évoquée plus haut. Dans son premier roman, où François Maspero déguise à peine l'histoire de sa famille pendant la Seconde Guerre mondiale, il livre ainsi quelques indices de la vision familiale de cette profession particulière:

Installé à la Valérane depuis le début de la guerre, il [le grand-père du Chat, personnage principal du roman] partageait son temps entre des activités foisonnantes et apparemment désordonnées. Celles-ci comprenaient d'abord la rédaction ou la mise à jour de ses manuels de médecine: il était toujours en retard et heureux de la distance, aggravée par la ligne de démarcation, qui le séparait de son éditeur, être mythique et chargé de toutes les irrationalités, voire de tous les ridicules, dont il stigmatisait l'affairisme et la rapacité supposés. «Espèce d'éditeur », grognait-il quand il surprenait l'un de ses petitsenfants en flagrant délit d'avarice, de chapardage louche ou d'étroitesse d'esprit; ou bien "je vais téléphoner à mon éditeur ", claironnait-il, lorsqu'il s'absentait un instant pour gagner les cabinets. La grand-mère du Chat brillait dans ses récits des réceptions des éditeurs, à Paris, avant la guerre, où l'on distinguait d'un simple coup d'œil ceux-ci des auteurs, à la mine florissante des uns, à l'air exsangue des autres. (Maspero 1998, 92)

Comment ne pas penser que François Maspero n'ait pas réellement entendu, dans la bouche de son grand-père Gaston, l'ironie et le sarcasme qui lui servaient à moquer les éditeurs? Ceux-ci étaient décrits comme appartenant à une engeance bassement intéressée, vénale et intellectuellement pauvre. Les auteurs et les écrivains, eux, étaient au contraire tout entier 
dévoués à leur vocation, ne récoltant jamais les profits qui venaient enrichir ceux qui les publiaient. Et la dernière phrase finit de poser l'image que François Maspero n'eut de cesse de vouloir démentir: le devoir de ne pas se reposer sur la réussite économique, la mise en place d'un modèle éditorial à l'opposé du "commerce» des grands éditeurs parisiens et, enfin, le refus et l'impossibilité de ne pas être au moins aussi «exsangue» que ses auteurs.

Parce qu'il s'est investi dans un métier déprécié du point de vue des "purs» intellectuels, tout en développant les qualités de «désintéressement » et d'engagement politique valorisées par ces derniers, François Maspero a recueilli une certaine reconnaissance. Le fait d'avoir tiré le métier d'éditeur et de libraire du côté de l'engagement politique a pu susciter une forme d'admiration de la part des personnes travaillant avec lui. Pourtant, on peut dire que ces deux dimensions montrent un rapport également antagoniste à l'égard de la famille. D'un côté en effet, l'édition est porteuse des stigmates de l'intéressement particulièrement décrié par le grand-père par exemple. De l'autre, l'élément politique tire le fil d'un certain héritage familial datant de l'expérience de la guerre. Dès lors, la position de François Maspero s'est construite comme une double subversion: subversion de la «loi» familiale par le choix d'un métier dévalué en son sein, d'une part, et, d'autre part, subversion de la «loi » de l'édition, domaine où le désintéressement doit généralement être pondéré par des objectifs de rentabilité économique.

Le charisme de François Maspero est né de la tension entre, d'un côté, des dispositions intellectuelles et politiques héritées et, de l'autre, leur investissement dans un métier astreint tout à la fois à des objectifs économiques et au désintéressement relatif des univers de production culturelle. En travaillant à faire valoir la dignité des militants révolutionnaires du monde, en leur octroyant un espace d'expression privilégié, voire unique, en fabriquant pour eux des livres qui eurent tous les attributs de la "grande» édition, l'éditeur François Maspero réconcilia, au cœur d'une profession ambivalente, l'exigence intellectuelle propre à ses ancêtres universitaires et l'éveil politique auquel il fut initié, dès son plus jeune âge, par son frère. Cependant, si un tel projet a entraîna un investissement entier et douloureux de la part de l'éditeur, la souffrance ne pouvait pas être légitimée, dans le cadre d'une entreprise «anti-capitaliste» et anti-paternaliste, par le charisme d'un individu spécifique. Elle trouvait sa place dans un système rationnel basé sur une justification politique: c'était au nom de la révolution 
(et non pas au nom d'une domination charismatique) qu'elle pouvait exister (sans être "souhaitée»). Ce système de justification correspond par ailleurs à une autre réalité douloureuse, une certaine forme de trahison, assumée, à l'égard d'une famille d'universitaires bourgeois. Mais cette trahison ne fut pas entière. C'est en investissant l'édition d'une dimension désintéressée et politique que François Maspero put faire valoir un héritage familial puissant, repoussant la possibilité d'exercer son métier sur un mode «facile», «léger» ou tout simplement «heureux» ${ }^{9}$.

\section{Références}

Aeschimann, E. (2007), «Tout reste à frère ", Libération, 7 août.

BARdoulllet, M.-C. (1977), La Librairie du travail, Paris, Maspero.

Clastres, P. (1974), La société contre l'État, Paris, Minuit.

KaLINOwSKI, I. (2005), Leçons wébériennes sur la science et la propagande, Marseille, Agone.

LiAuZu, C. (1991), «Intellectuels du tiers monde et intellectuels français. Les années algériennes des éditions Maspero ", dans J. P. Rıoux et J.-F. SIRINELLI, dir., La Guerre d'Algérie et les intellectuels français, Bruxelles, Complexe, p. 155-174.

MARKer, C. (1971), Maspero: Les mots ont un sens, Paris, Iskra.

Maspero, F. (1998) [1984], Le sourire du chat, Paris, Seuil. (2002), Les abeilles et la guêpe, Paris, Seuil.

NoIRIel, G. (2005), Les fils maudits de la République, Paris, Fayard.

Péguy, C. (1947), Péguy et les Cahiers. Textes concernant la gérance des Cahiers de la quinzaine, Paris, Gallimard (Bibliothèque de la Pléiade).

Simonin, A. (1991), «Les éditions de Minuit et les éditions du Seuil. Deux stratégies éditoriales face à la Guerre d'Algérie », dans J. P. Rıoux et J.F. SIRINELLI, dir., La Guerre d'Algérie et les intellectuels français, Bruxelles, Complexe, p. 219-245.

Weber, M. (1995) [1971], Économie et société, t.1, Paris, Plon. (2006), Sociologie de la religion, Paris, Flammarion.

9. Je remercie Isabelle Kalinowski, dont les remarques et l'attention autour de ce texte m’ont été très précieuses. 


\section{Résumé}

Centré sur le personnage de l'éditeur français François Maspero, cet article traite des liens entre le charisme et la souffrance de l'individu charismatique. Il met en perspective d'un côté l'existence et la manifestation de la domination charismatique dans le contexte politique des années 1960 et 1970 et, de l'autre côté, l'investissement personnel extrêmement appuyé dans le travail, débouchant sur un rapport douloureux et contraignant à celui-ci. Loin d'être la conséquence d'un mode d'exercice du métier, la souffrance doit plutôt être rapportée aux motivations biographiques et à l'héritage familial à l'origine du choix de l'investissement dans un métier, l'édition, sur un modèle contestataire.

\section{Abstract}

By focusing on the character of the French publisher François Maspero, this article examines the relation between charisma and suffering as it is experienced by the charismatic. In the political context of the 1960s and the 1970s, it is necessary to question at the same time the existence and manifestation of the charismatic domination on one hand, and on the other hand the very strong personal investment in work, making it painful and rather distressing. Far from being a consequence of such a relationship to work, suffering must rather be thought of in relation to the family heritage which may explain the choice of a profession - publisher- fulfilled in a politically radical way. 